\title{
Genetic and genomic approaches to understanding drug resistance in parasites
}

\author{
EILEEN DEVANEY* \\ Institute of Infection Immunity and Inflammation, University of Glasgow, Bearsden Road, Glasgow G61 1QH
}

(Received 14 Fune 2013; accepted 16 Fune 2013)

Drug resistance is a major challenge in many infectious diseases including those caused by parasites. In the absence of vaccines, treatment and control of most parasitic diseases will continue to rely almost exclusively on the use of drugs for the foreseeable future. Until recently, the paucity of new drugs for many parasites coupled with the inexorable spread of resistance heralded a potentially catastrophic situation, as exemplified by significant anthelmintic resistance in many parasitic nematodes of veterinary significance. Times may be changing, however; the London declaration demonstrated a new commitment from the pharmaceutical industry at least for neglected tropical diseases (http://www. unitingtocombatntds.org/), while the increasing availability of genetic and genomic resources for many parasites should facilitate the identification of genes involved in resistance and suggest novel approaches to managing this problem.

Drug resistance is defined as a heritable reduction in the sensitivity of a parasite population to the action of a drug. There are many good reasons for attempting to understand the genetic basis of resistance: for the development of sensitive and specific markers to identify and track drug-resistant parasites in field populations, to facilitate studies aimed at understanding the origin and spread of resistance, to determine whether the resistant phenotype can be manipulated or reversed, to better understand the mode of action of some drugs, and for assessing programmes/treatment schedules aimed at minimizing the spread of resistance. Furthermore, if drug resistance could be detected early it might be possible to eliminate parasites resistant to one drug before the resistant genotype prevails. Given that the treatment of most parasitic infections relies on a very limited pharmacopoeia, preserving the life-span of

\footnotetext{
* Corresponding author. Institute of Infection Immunity and Inflammation, University of Glasgow, Bearsden Road, Glasgow G61 1QH, UK. Tel: + 4401413306925. Fax: + 440141330 2271. E-mail: eileen.devaney@glasgow. ac.uk
}

existing drugs in the face of increasing levels of resistance is of great importance.

In this Special Issue of Parasitology, we bring together seven reviews covering various approaches to understanding mechanisms of drug resistance in some of the major parasite species of humans and animals. Some common themes emerge in these papers: an important question raised by several authors is how to distinguish treatment failures from true resistance. Treatment failures can arise from many factors other than resistance, including variation in the strain or isolate of parasite under study, variation in host pharmacokinetics, variation in the susceptibility of different life-cycle stages to drug, the impact of infection intensity, the source and activity of the drug, as well as factors relating to the suitability and sensitivity of methods used to assess drug efficacy. These issues only serve to stress the desirability of developing molecular probes to assess properly the occurrence and extent of resistance. Other common themes include: the pros and cons of the 'candidate gene' approach. Many previous studies on the mechanisms of drug resistance in various parasite species focused largely on the identification of mutations or single nucleotide polymorphisms (SNPs) in genes which were predicted either to be drug targets or to be involved in the uptake, metabolism or efflux of drugs. It is now appreciated that, at least in some systems, the basis of resistance is likely to be multigenic, highlighting the desirability of adopting genome-wide approaches. The new tools being developed such as the various 'omics' approaches and the genetic and genomic resources becoming available for many parasite species will allow non-assumptive approaches to be adopted to identify the genetic basis of resistance based on genome-wide analysis.

Given the continuing devastating impact of malaria, particularly in sub-Saharan Africa, the issue of drug resistance remains a priority for research. We start with a paper on Plasmodium falciparum (Mwangi and Ranford-Cartwright, 2013), which presents an historical overview of anti-malarial drug development and the spread of resistance. Chloroquine was 
first introduced in the 1940s, and resistance in $P$. falciparum first reported in the 1960 s. Since then chloroquine resistance has spread, so that it now more or less mirrors the distribution of the parasite. While polymorphisms in specific genes are associated with resistance to chloroquine, there is still debate as to how resistance arises and whether additional alleles are involved. Combination therapies are generally considered to slow down the rate of development of drug resistance, but the use of artemisinin-based combination therapies, the first-line treatment for uncomplicated $P$. falciparum in many regions of the world, is now threatened by the emergence of artemisinin resistance in South East Asia. Recent studies on artemisinin resistance highlight novel mechanisms by which parasites can tolerate drug pressure, such as the apparent ability of ring-stage parasites to enter a quiescent state in the presence of drug (Witkowski et al. 2010). This interesting phenotype helps explain the delayed clearance rate of parasites, a characteristic of artemisinin resistance.

Vector control via spraying and the use of insecticide-treated bed nets has had a major impact on the disease burden attributed to malaria in the past 20 years. Unfortunately, just as resistance is widespread to anti-malarial drugs, the same is true for insecticide resistance in field populations of malaria vectors. Witzig and colleagues (2013) deal with this subject in their paper on pyrethroid resistance in Anopheles gambiae. Their contribution highlights many of the issues surrounding the identification of loci involved in resistance: candidate gene approaches have been successfully applied to $A$. gambiae, while the availability of the genome has identified a large number of SNPs that may be associated with resistance. They describe the use of genetic crosses between natural field populations (rather than laboratory colonies) of $A$. gambiae to map quantitative trait loci (QTLs) associated with insecticide resistance and highlight the pros and cons of the various approaches to identifying genes underlying pyrethroid resistance.

In their contribution, Alsford et al. (2013) describe the limitations of the drugs currently available for treatment of human African trypanosomiasis (HAT) and Chagas disease, two parasitic infections with very limited options for chemotherapy. The toxicity of many of the drugs used to treat HAT impacts on patient compliance and makes defining resistance, rather than treatment failure, challenging. Much of the literature on drug resistance in African trypanosomes revolves around candidate genes such as drug transporters, which are known to be altered in some drug-resistant isolates of Trypanosoma brucei. More recent efforts to identify genes involved in drug resistance in trypanosomes have used genome-wide RNAi approaches. By introducing several modifications to the standard RNAi methodology, it has been possible to develop an inducible high- throughput RNAi screen followed by next generation sequencing (RIT-seq for RNA interference target sequencing) to detect $T$. brucei parasites able to survive drug treatment. This approach identifies loss-of-function mechanisms that confer resistance to the parasite. These studies confirmed the role of drug transporters in resistance to specific drugs, while also identifying a number of novel factors, some of which would not have otherwise been implicated in resistance.

Finally for the protozoan parasites, Berg et al. (2013) describe a numbers of 'omics' approaches to identifying mechanisms of drug resistance in the Leishmania species. Given the small number of drugs available to treat leishmaniasis, their review stresses the desirability of safeguarding the few available compounds. Leishmania species adopt multiple mechanisms to survive drug exposure including sequence polymorphisms in specific genes and other modifications, such as gene amplification and variation in copy number. In addition, as intra-cellular parasites in the mammalian host, resistance to antimonial compounds can also involve modification of host cell genes as well as parasite genes. They summarize recent data from metabolomics studies and argue for the integration of these approaches with genomic studies. Importantly, they end with a discussion of how data arising from such high tech experiments can be translated to the field in resource-poor settings.

The next two articles by Gilleard (2013) and Hodgkinson et al. (2013) deal with the ongoing issues of drug resistance in helminths of veterinary significance. Here, nematodes such as Haemonchus contortus and Teladorsagia circumcincta give the greatest cause for concern, as resistance is prevalent to all three classes of widely used anthelmintic. The inability of anthelmintics to control veterinary species, such as Haemonchus, threatens the viability of the livestock industry in some parts of the world. As the same drugs are also used in mass drug administration (MDA) programmes for human diseases, such as hookworm infection and onchocerciasis, this inevitably gives rise to concern as to the long-term sustainability of such approaches, as highlighted by the failure of ivermectin treatment in Onchocerca volvulus (Osei-Atweneboana et al. 2011). As reviewed by Gilleard (2013), research on parasitic nematodes has suffered from a lack of genetic and genomic resources, a situation that is beginning to change with the availability of genomic and transcriptomic sequences from species such as Haemonchus. In addition, the experimental tractability of $H$. contortus, together with a number of novel resources such as the genetic backcrosses between well-defined strains of differing drug sensitivity, demonstrate many of the advantages of using Haemonchus as an experimental model. In his article, Gilleard also highlights the desirability of monitoring the integrity of parasite isolates used in different 
laboratories around the world, an important but often over-looked issue when comparing data from a variety of laboratories. Forward genetic approaches, such as the backcross lines in which resistance genes have been introgressed into a susceptible genetic background and taken through successive generations of crossing with ivermectin selection, are already providing much useful data. Using microsatellite loci, it was shown that the backcross progeny were genotypically similar to the susceptible parent, but phenotypically resistant to ivermectin. A better understanding of issues such as population structure and size, together with the continued generation of tools such as microsatellite markers and SNPs, all contribute to the potential to undertake population genetic studies in Haemonchus.

A similar approach has recently been adopted for the liver fluke Fasciola hepatica, a parasite with an increasing distribution due to climate change and livestock movement. As with the other parasites dealt with in this issue, there are few drugs available for the treatment of liver fluke and resistance to triclabendazole (TCB), the most widely used of these, is an increasing problem. The article by Hodgkinson and colleagues (2013) reviews the current status of TCB resistance and summarizes the availability of genetic resources for $F$. hepatica research. A forward genetic approach to identifying genes involved in TCB resistance has been adopted by the authors, based on the generation of a backcross between TCBsusceptible and TCB-resistant parasites. Taking advantage of the life-cycle of $F$. hepatica, in which clonal expansion takes place in the snail host, lines were generated from confirmed TCB-susceptible and -resistant isolates from $\mathrm{UK}$ field populations, the aim being to generate $\mathrm{F} 1$ and $\mathrm{F} 2$ recombinants for genetic mapping. A SNP discovery programme and the identification of neutral microsatellite markers from the draft genome sequence will facilitate the identification of recombinants and, in future studies, regions of the genome under TCB selection.

The final article by Greenberg (2013) deals with the spectre of praziquantel resistance in schistosomes. In common with many other helminth parasites of humans, chemotherapy is based largely on a single drug, in this case praziquantel, which is frequently delivered via MDA campaigns. Praziquantel shows activity against all species of human schistosome and is relatively inexpensive, and thus is the drug of choice for control and treatment of this infection. The degree of resistance to praziquantel in field population of schistosomes remains the subject of much debate, but there are well-documented cases of treatment failures and resistant parasites have been selected in laboratory studies. The widespread dependency on praziquantel coupled with the fact that there are few alternative drugs currently on the market gives cause for concern. Like many other anthelmintics, the precise mode of action of praziquantel on schistosomes remains unresolved.
Greenberg provides a useful update on the status of research on drug efflux mechanisms, such as the expression of multi-drug transporters and their possible association with praziquantel resistance in flukes, including Schistosoma mansoni. The article concludes with an overview of the genetic toolkit now available to address issues such as drug resistance: the genome sequences for all three species of human schistosome, the transcriptome data for S. mansoni, the availability of a genetic map for $S$. mansoni, the ability to undertake genetic crosses, the application of RNAi and transgenesis together set the scene for major advances in the field of schistosome biology.

As is clear from these articles, the scientific community has been extremely resourceful in developing and applying new tools to address the problem of drug resistance. Technology is on our side, with the availability and affordability of massively parallel (or next generation) sequencing, the construction of genetic maps for several important parasite species and a variety of genome wide approaches, which should allow the identification of areas of the genome with signatures of drug selection. In the future, the emphasis will shift from studies on laboratory isolates to population genetic studies, such as that recently reported for $P$. falciparum. Here regions of the genome under selection following artemisinin therapy were identified using genome-wide approaches (Cheeseman et al. 2012). For metazoan parasites with large genomes, techniques such as Restriction-site Associated DNA Sequencing (RADSeq), a method of reduced representation genome sequencing (Davey et al. 2011), can be applied to the genotyping of field populations of known drug sensitivity. While advances in technology offer enormous opportunities, the success of such methods is highly dependent on having well-characterized biological material of known phenotype in relation to drug resistance. Ultimately, the application of genetic and genomic tools to the problem of drug resistance will provide a better understanding of the evolution and spread of resistance alleles within populations. These data will be important for designing and monitoring programmes aimed at restricting the spread of resistance and preserving the life-span of the few existing drugs, until alternative control strategies are available.

\section{ACKNOWLEDGEMENTS}

ED would like to acknowledge Dr Roz Laing and Emeritus Professor Andy Tait, University of Glasgow for their comments on the manuscript and for many stimulating discussions.

\section{REFERENCES}

Alsford, S., Kelly, J. M., Baker, N. and Horn, D. (2013). Genetic dissection of drug resistance in trypanosomes. Parasitology 140, 1478-1491. Berg, M., Mannaert, A., Vanaerschot, M., Van Der Auwera, G. and Dujardin, J.-C. (2013). (Post-) Genomic approaches to tackle drug resistance in Leishmania. Parasitology 140, 1492-1505. 
Cheeseman, I. H., Miller, B.A., Nair, Nkhoma, S., Tan, A., A1 Saai, S., Phyo, A. P., Moo, C. L., Lwin, K. M., McGready, R., Ashley, E., Imwong, M., Stepniewska, K., Yi, P., Dondorp, A. M., Mayxay, M., Newton, P. N., White, N.J., Nosten, F., Ferdig, M. T. and Anderson, T.J. (2012). A major genome region underlying artemisinin resistance in malaria. Science 336, 79-82. doi: 10.1126/ science. 1215966

Davey, J. W., Hohenlohe, P. A., Etter, P. D., Boone, J. Q. Catchen, J. M. and Blaxter, M. L. (2011). Genome-wide genetic marker discovery and genotyping using next-generation sequencing. Nature Reviews Genetics 12, 499-510. doi: 10.1038/nrg3012.

Gilleard, J. S. (2013). Haemonchus contortus as a paradigm and model to study anthelmintic drug resistance. Parasitology 140, 1506-1522.

Greenberg, R. M. (2013). New approaches for understanding mechanisms of drug resistance in schistosomes. Parasitology 140, 1534-1546.

Hodgkinson, J., Cwiklinski, K., Beesley, N. J., Paterson, S. and Williams, D. J. L. (2013). Identification of putative markers of tricalbendazole resistance by a genome-wide analysis of genetically recombinant Fasciola hepatica. Parasitology 140, 1523-1533.

Mwangi, J. M. and Ranford-Cartwright, L. C. (2013). Genetic and genomic approaches for the discovery of parasite genes involved in antimalarial drug resistance. Parasitology 140, 1455-1467.

Osei-Atweneboana, M. Y., Awadzi, K., Attah, S. K., Boakye, D. A., Gyapong, J. O. and Prichard, R. K. (2011). Phenotypic evidence of emerging ivermectin resistance in Onchocerca volvulus. PLoS Neglected Tropical Diseases 5, e998. doi: 10.1371/ journal.pntd.0000998

Witkowski, B., Lelièvre, J., Barragán, M. J., Laurent, V., Su, X. Z., Berry, A. and Benoit-Vical, F. (2010). Increased tolerance to artemisinin in Plasmodium falciparum is mediated by a quiescence mechanism. Antimicrobial Agents and Chemotherapy 54, 1872-1877. doi: 10.1128/ AAC.01636-09.

Witzig, C., Wondji, C.S., Strode, C., Djouaka, R. and Ranson, H. (2013). Identifying permethrin resistance loci in malaria vectors by genetic mapping. Parasitology 140, 1468-1477. 